\title{
The Effect of Aging and Cardiac Disease on that Portion of QT Interval Variability that Is Independent of Heart Rate Variability
}

\author{
V Starc $^{1}$, TT Schlegel ${ }^{2}$ \\ ${ }^{1}$ Faculty of Medicine, University of Ljubljana , Ljubljana, Slovenia \\ ${ }^{2}$ NASA-Johnson Space Center, Houston, TX USA
}

\begin{abstract}
Our goal was to remove all readily explainable (extrinsic) portions of beat-to-beat $Q T$ interval variability $(Q T V)$ from total QTV so as to derive the purest possible measure of intrinsic QTV. For this purpose we developed a model that describes extrinsic QTV based on other factors ascertainable from the ECG, such as changes in $R R$ intervals, voltage amplitudes and QRS-T angles. Five-min supine high-fidelity 12-lead ECGs were acquired from 101 individuals, including from 20 healthy young men (age 21 \pm 1 ) and from three groups of middleaged individuals (52 \pm 11 years) with increasing levels of cardiac disease. By fitting our model data to the measured QT interval signal, we calculated the explained (extrinsic) part of QTV, and the difference between the measured and the calculated QT signal represented the "unexplained" (or intrinsic) part of QTV. Results suggest that the ratio of unexplained to explained QTV increases with both age and cardiac disease.
\end{abstract}

\section{Introduction}

The increasing use of the so-called "QT interval variability index" (QTVI) [1] reflects the fact that beatto-beat QT interval variability (QTV) is heavily influenced by the simultaneous RR interval variability (RRV). In this study, we isolated that part of QTV that can be readily explained by RRV and/or by other extrinsic factors ascertainable from the ECG, such as, for example, the changes in voltage amplitude (Va) and QRS-T angle ( $\mathrm{RTa}$ ) occurring with respiration.

Our goal was to remove all readily explainable portions of QTV from total QTV so as to derive the purest possible measure of intrinsic QTV. For this purpose we used a model with a fast component that varies linearly with the RR interval, RTa and Va signals, and also a slow component describing QT interval dynamics that decays to a steady QT value that depends on RR. By fitting our model data to the measured QT interval signal we calculated the explained part of QTV, and the difference between the measured and the calculated QT signal in a given time window represented the "unexplained" (or intrinsic) part of the signal.

\section{Methods}

\subsection{A model for determination of the explained and unexplained part of QTV}

Let the QT interval be a function of the RR interval, Va signal, RTa signal and time,

$$
Q T=Q T(R R, V a, R T a, t)
$$

where $\mathrm{Va}$ is a voltage amplitude represented by the series of the first principal eigenvalue of the QRS complex, and $\mathrm{RTa}$ is a series of QRS-T angles represented by the angle between the first principal axes of the QRS complex and the T wave, respectively, all obtained for each ECG beat.

Since the RR interval, Va and RTa signals are not independent, their mutual relationship can be written as

$$
\begin{aligned}
& V a=V a(R R), \\
& R T a=R T a(R R, V a) .
\end{aligned}
$$

A change in QT interval driven by other influences observable on the ECG can be written as a sum of all proposed influences,

$$
d Q T=d Q T_{R R}+d Q T_{V a}+d Q T_{R T a}+d Q T_{t}
$$

where each subscript denotes the contribution of each independent signal to QTV, RR interval, voltage amplitude $\mathrm{Va}$, angle $\mathrm{RTa}$, and time representing dynamic properties of QT interval, respectively. dQT can be further represented as

$$
d Q T=\alpha \cdot \partial R R+\beta \cdot \partial V a+\gamma \cdot \partial R T a+(d Q T / t) d t
$$

with $\alpha, \beta$, and $\gamma$ as partial derivatives $(\alpha=\partial Q T / \partial R R$, $\beta=\partial Q T / \partial V a$ and $\gamma=\partial Q T / \partial R T a)$ and $\partial \mathrm{RR}, \partial \mathrm{Va}$, and $\partial \mathrm{RTa}$ as the corresponding differentials.

Next, assume that the time dependence is described as a first order process with QT decaying to a steady QTc value, depending on the RR interval only,

$$
Q T_{c}=Q T_{0}\left(R R / R R_{0}\right)^{J}
$$




$$
d Q T / d t=-\left(Q T-Q T_{c}\right) / \tau
$$

where $\tau$ is a time constant estimated as $55 \mathrm{~s}$ [2].

Since RR interval is an independent variable, dRR, which can be directly measured, can be substituted for $\partial R R$ in (5). dRR is specifically the change in RR interval between the two consecutive beats, whereas $\mathrm{dt}$ is the time from one beat to another, which equals the duration of the following $R R$ interval, $R R_{i+1}$.

On the other hand, differentials $\partial \mathrm{Va}$ and $\partial \mathrm{RTa}$ can be obtained the following way. The signals $\mathrm{Va}$ and $\mathrm{RR}$ are related by (2), hence the total differential can be expressed as,

$$
d V a=\xi \cdot \partial R R+\partial V a,
$$

where $\xi=\partial V a / \partial R R$. The first part of (8) represents change in dVa due to change in RR and the last part the change independent of RR.

Next, the signals RTa, Va and RR are related by (3), hence the total differential can be expressed as

$$
d R T a=\eta \cdot \partial R R+\vartheta \cdot \partial V a+\partial R T a,
$$

where $\eta=\partial R T a / \partial R R$, and $\vartheta=\partial R T a / \partial V a$.

After eliminating the corresponding partial differentials from (5) using (8) and (9), the final relationship can be written as

$$
\begin{aligned}
& d Q T=\alpha \cdot d R R+\beta \cdot(d V a-\xi d R R)+ \\
& +\gamma \cdot(d R T a-\eta d R R-\vartheta d V a)-\left(Q T-Q T_{c}\right) d t / \tau
\end{aligned}
$$

Whereas dQT, dRR, dVa and dRTa are measured quantities, all partial derivatives representing constant parameters can be determined in the following way. First, $\xi$, the slope between Va and RR, as given by (8), can be determined from a given data set of $\mathrm{Va}$ and $\mathrm{RR}$ of a given time window using the least square method. Next, the parameters $\eta$ and $\theta$, as given by (9), can be determined from a given data set of RTa, Va and RR signals and the parameter $\xi$ for the same time window. With $\xi$, $\eta$, and $\theta$ known, and with an initial approximation of $\mathrm{QT}_{0}$ and the exponent $\mathrm{J}$, the least square method is again applied to (10) to determine the remaining parameters $\alpha, \beta$, and $\gamma$.

Since the $\mathrm{QT}_{0}$ and $\mathrm{J}$ are only approximate, they are determined using a larger time window with up to 256 beats. Starting with the initial approximation of $\mathrm{QT}_{0}$ and $\mathrm{J}$, parameters $\alpha, \beta, \gamma, \xi, \eta$, and $\theta$, are determined for each median beat within a window containing 32 beats, ultimately covering a larger window of 256 beats. By moving a 32 beat window within the 256 beat window, the signals $\alpha(\mathrm{t}), \beta(\mathrm{t}), \gamma(\mathrm{t}), \xi(\mathrm{t}), \eta(\mathrm{t})$, and $\theta(\mathrm{t})$ that change with time are obtained. Then, (10) is integrated in time to calculate the time course of the explained part of QT. Finally, the difference between the measured and the calculated QT interval is squared and summed over all beats of the larger time window to represent the error signal
$\chi^{2}=\sum_{i}\left(Q T_{i, \text { measured }}-Q T_{i, \text { calculated }}\right)^{2}$,

which is minimized to obtain the optimal $\mathrm{QT}_{0}$ and $\mathrm{J}$ for the entire larger window.

The procedure is repeated to provide new series of $\alpha$, $\beta, \gamma, \xi, \eta$, and $\theta$ signals, and also more and more accurate $\mathrm{QT}_{0}$ and $\mathrm{J}$ parameters.

The error signal normalized with the number of beats then represents the amount of the QT change that cannot be described by RR, Va or RTa signals. When this is divided by the sum of the total QT variance, it represents the unexplained part of the QTV (uQTV). QTV is specifically quantified as the standard deviation of normal-to-normal (SDNN) QT intervals in the first eigenvector [3], with the explained QTV (eQTV) being equal to the total QTV minus the unexplained QTV (uQTV). The ratio of unexplained to explained QTV is further quantified logarithmically as an "index of unexplained QTV", or IUQTV, specifically as $\log (\mathrm{uQTV} / \mathrm{eQTV})$.

In our method, we do not divide the cumulative error signal with the variance, but rather use the integrated absolute signal difference, divided by the integrated absolute dQT signal. When properly normalized, the time series of $\alpha, \beta$, and $\gamma$ provide the contribution of each signal to the QTV.

\subsection{Patients and data acquisition}

Five-min supine high-fidelity 12-lead ECGs were

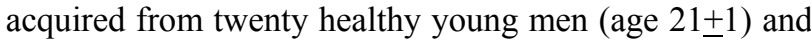
from twenty-seven individuals in each of the three following groups matched for both age $(52 \pm 11,57 \pm 9$, and $52 \pm 13$ years, respectively) and gender $(15 \mathrm{M}, 12 \mathrm{~F})$ : 1) asymptomatic middle-aged persons; 2) patients with known coronary artery disease (CAD) but normal left ventricular ejection fraction (LVEF); and 3) patients with cardiomyopathy (CMP) and known low LVEF $(<40 \%)$. QT interval and other related quantities were determined using methods as described earlier [3]. Our model was thereafter utilized to calculate the eQTV, uQTV and IUQTV, respectively, as described above.

\section{Results}

Mean \pm SD values for the IUQTV were $-0.03 \pm 0.25$ for the young male group, $0.09 \pm 0.26$ for the asymptomatic middle-aged group, $0.22 \pm 0.27$ for the $\mathrm{CAD}$ group $(\mathrm{P}<0.01$ vs. the young male group) and $0.25 \pm 0.25$ for the CMP group $(\mathrm{P}<0.001$ vs. the young male group and $\mathrm{P}<0.05$ vs. the asymptomatic middle-aged group). Only in the young men did the explained part of QTV usually exceed $50 \%$ of total QTV, thus making $\log (\mathrm{uQTV} / \mathrm{eQTV})$ most often a negative number. Figure 1 below provides and example of QT interval dynamics in a healthy 
middle-aged person.

Healthy middle aged person
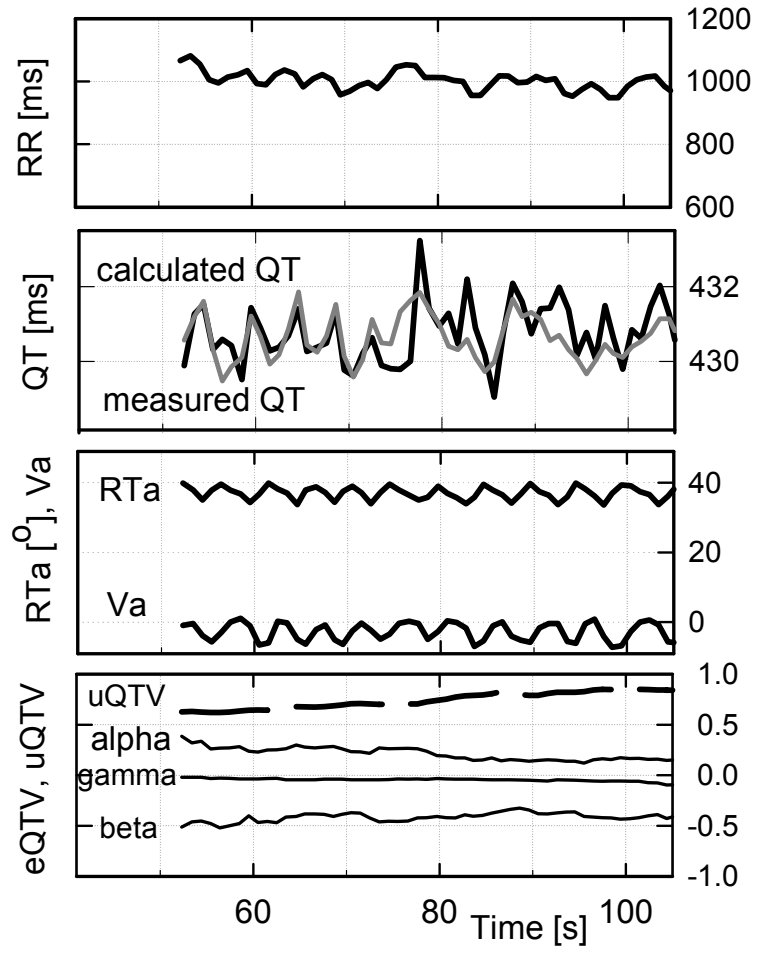

Figure 1. QT interval dynamics in a healthy middleaged person. (See text for abbreviations). The panels show, from top to the bottom, the time courses of the RR interval, measured and calculated QT interval, RTa, Va (relative units), and lastly eQTV and uQTV. The eQTV is represented by time courses of the parameters alpha, beta, and gamma for independent RR, Va and RTa changes, respectively. These are normalized to the total QTV. The uQTV is low when the QT interval course can be mostly reconstructed by the independent variables $\mathrm{RR}, \mathrm{Va}$ and RTa. It approaches 1 when the QT interval course has little dependence on the above variables.

\section{Discussion and conclusions}

Our study showed that QT interval changes can be partially reconstructed by a model that considers a fast component of QT interval change that varies linearly with the RR, RTa and Va signals, and also a slow component that depends on the RR signals and that decays to a steady QT value. The model takes into account the hysteresis-like properties of QT interval dynamics [4] and also the fact that while changes in QT intervals are predominantly driven by changes in RR intervals [5], they can also occur in response to changes in QT wavefront direction descriptors, such as in the QRS-T angle or equivalent $[6,7]$. Although changes in ECG amplitudes other than that of the QRS complex, for example in that of the $\mathrm{T}$ wave, are also capable of driving QT interval changes and may be suitable for incorporation into future iterations of the model, we did not incorporate T-wave amplitude changes into our initial model in order to avoid the effects of potentially confounding mid-wave fluctuations in $\mathrm{T}$ wave morphology. In the practical sense, the results of our clinical study suggest that the ratio of unexplained to explained QTV (i.e., the IUQTV) increases with both age and cardiac disease. However, the relative diagnostic and prognostic value of the IUQTV in comparison to QTVI remains to be determined.

\section{Acknowledgements}

Supported by Grant No. P3-0019, Ministry for Higher Education, Science and Technology and, Slovenia.

\section{References}

[1] Piccirillo G, Magri D, Matera S, et al. QT variability strongly predicts sudden cardiac death in asymptomatic subjects with mild or moderate left ventricular systolic dysfunction: a prospective study. Eur Heart J 2007;28:1344-1350.

[2] Franz MR, Swerdlow CD, Liem LB, and Schaefer J. Cycle length dependence of human action potential duration in vivo. J Clin Invest 1988; 82: 972-979

[3] Starc V, Schlegel TT: Real-time multichannel system for beat-to-beat QT interval variability. J Electrocardiol 39:358-67, 2006.

[4] Lang CCE, Flapan AD, Neilson JMM. The impact of QT lag compensation on dynamic assessment of ventricular repolarization: reproducibility and the impact of lead selection. PACE 2001; 24: 366-373.

[5] Almeida R, Gouveia S, Rocha AP, Pueyo E, Martinez JP, Laguna P. QT variability and HRV interactions in ECG: quantification and reliability. IEEE Trans Biomed Eng. 2006;53:1317-1329.

[6] Acar B, Yi G, Hnatkova K, Malik M. Spatial, temporal and wavefront direction characteristics of 12-lead T-wave morphology. Med Biol Eng Comput 1997; 37: 574-584.

[7] Kors JA, van Herpen G, Bemmel JH. QT dispersion as an attribute of T-loop morphology. Circulation 1999; 99: 1458-1463.

Address for correspondence

Vito Starc

Faculty of Medicine,

University of Ljubljana

Zaloška 4,

1000 Ljubljana, Slovenia

vito.starc@mf.uni-lj.si 